\title{
Research on stamping die reconstruction method based on binocular stereo vision
}

\author{
Chen Sisi $^{12, \text { a }}$, Li Zhanguo ${ }^{12, b}$, Shi Yaochen ${ }^{2, \mathrm{c}}$ and Cai Yunguang ${ }^{2, \mathrm{~d}}$ \\ ${ }^{1}$ Changchun University of Technology,Changchun,130012,China \\ ${ }^{2}$ Changchun University, Changchun, 130022, China \\ a250793721@qq.com, b461666473@qq.com, ’shiyaochen126@126.com, d279309014@qq.com
}

Keywords: free-form surface, multiple views together, surface reconstruction.

Abstract. In this paper, by studying the principle of binocular stereo vision $3 \mathrm{~d}$ scanning imaging and scanning data splicing method. The author uses the binocular stereo vision method make a 3D scanning for parts and use NURBS curve reconstruction technology for reverse design of parts.

\section{Introduction}

Reverse engineering are opposite to the traditional design process, form the product model recover engineering design, and used for engineering analysis and innovation. Reverse engineering can shorten the design cycle and improve design efficiency. Therefore reverse engineering is widely used in 3D measurement and parts repair of automobile, aviation, machinery, medicine, art and other industries.

\section{Reverse engineering}

Reverse engineering technology is in the absence of design drawings, In the absence of design drawings of the circumstances, through the three-dimensional measurement, data pretreatment and curve surface fitting techniques to reconstruct the part of the three-dimensional model. The stamping die parts as shown in figure 1,The parts is mainly composed of free-form surface, positioning pin and the bolt hole, the free surface affect the precision of the workpiece precision, position precision of positioning pin and a link bolt hole directly affect the installation precision of workpiece. In order to reverse design of the part, binocular stereo vision $3 \mathrm{~d}$ scanning must be apply to it. Binocular stereo vision inspection technology uses two fixed image sampling unit (camera) acquire part's image from different angles and apply the parallax principle to image processing to restore the $3 \mathrm{D}$ geometry information of components.

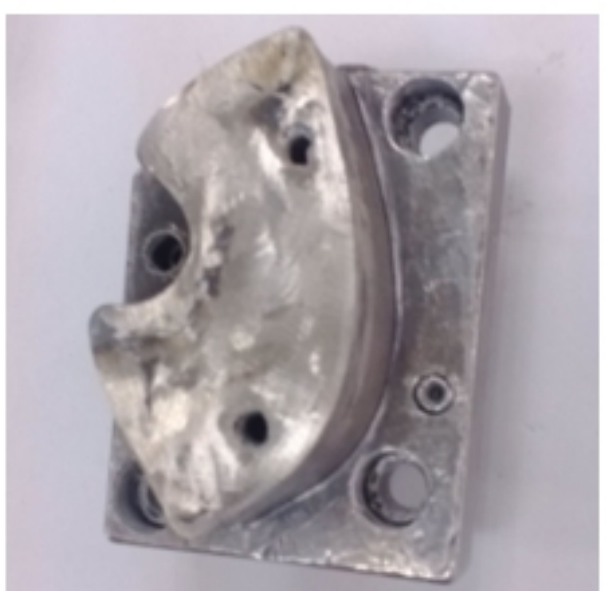

Figure1 the stamping mould

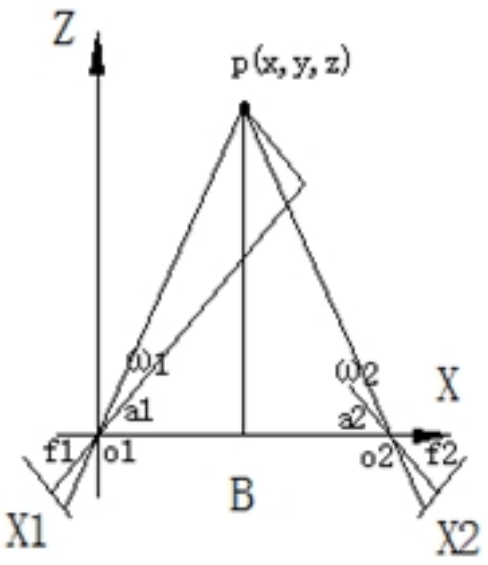

Figure2 concurrent structure 


\section{The principle of binocular stereo vision}

According to the relative position of the image acquisition unit, binocular stereo vision technology is divided into parallel structure and aggregation structure. As shown in Figure 2.Note that two kinds of structures are ideal model, also is the the pinhole model, this model ignores the lens induced by nonlinear distortion, CCD quantization effect etc.

Using the camera collection structure,$\left(x_{l}, y_{l}\right)$ is coordinate which measured by the left of camera through the measured object point, $f_{l}$ is the focal distance which came from the left camera, $R=\left[\begin{array}{ccc}r_{1} & r_{2} & r_{3} \\ r_{4} & r_{5} & r_{6} \\ r_{7} & r_{8} & r_{9}\end{array}\right]$ is a rotation matrix which producted by the interaction of two cameras, $T=\left[\begin{array}{c}t_{x} \\ t_{y} \\ t_{z}\end{array}\right]$ is a translation matrix which producted by the interaction of two cameras. Thus, the object's three-dimensional coordinates as formula(1) shown:

$$
\begin{aligned}
& x=z x_{l} / f_{l} \\
& y=z y_{l} / f_{l} \\
& z=\frac{f_{l}\left(f_{r} t_{x}-x_{l} t_{z}\right)}{x_{l}\left(r_{7} x_{l}+r_{8} y_{l}+r_{9} f_{l}\right)-f_{r}\left(r_{1} x_{l}+r_{2} y_{l}+r_{3} f_{l}\right)}
\end{aligned}
$$

Through the formula we can understand that, if known baseline distance, focal length and camera distance ,3D coordinates of the object points can be calculated.

\section{Multi view registration}

Because the measured workpiece is a 3D whole which composed by multiple surface, while the CCD camera imaging from a perspective of regional, so the shielding phenomenon can not be avoided .This block can be divided into: shape occlusion, curvature occlusion etc. Usually, people increase the collection angle to solve the occlusion phenomenon in 3D scanning process and the entire part 's three dimensional morphology was obtained by many times data collect. The essence of data splicing algorithm is transform data's coordinate, the data of PI through the rotation transformation matrix $\mathrm{R}$ and the translation transformation matrix $\mathrm{T}$ moving to the target position pi*, marked as:

$$
p_{i}^{*}=p_{i} \bullet R+T
$$

Although position transform of the mark point is rigid transformation in the process of multi view registration, there is a error in the process of actual measurement among two groups of mark point data couldn't completely correct splicing together. Considering the errors, the expression can improved as:

$$
p_{i}^{*}=p_{i} \bullet R+T+\delta_{i}
$$

To obtain the most appropriate location state, the least square method can be used and the least squares function as follows:

$$
F=\sum_{i=1}^{3}\left\|p_{i}^{*}-\left(p_{i} \bullet R+T\right)\right\|^{2}
$$

)

Assuming the solutions of $\bar{R}$ and $\bar{T}$ to satisfy the least squares function, so $p_{i}^{\prime}=p_{i} \bullet \bar{R}+\bar{T}$ and $p_{i}^{*}$ have the same centroid, among them: $c=\frac{1}{3}\left(p_{1}+p_{2}+p_{3}\right)$ is the quality center of $p_{i}$; 
$c^{\prime}=\frac{1}{3}\left(p_{1}^{\prime}+p_{2}^{\prime}+p_{3}{ }^{\prime}\right)$ is the quality center of $p_{i}^{\prime} ; c^{*}=\frac{1}{3}\left(p^{*}{ }_{1}+p^{*}{ }_{2}+p^{*}{ }_{3}\right)$ is the quality center of $p_{i}^{*}$; if $d_{i}=p_{i}-c_{;} d^{*}{ }_{i}=p^{*}{ }_{i}-c^{*}$, the target function could be rewrited as:

$$
F=\sum_{i=1}^{3}\left\|d_{i}^{*}-R d_{i}\right\|^{2}=\sum_{i=1}^{3}\left(d_{i}^{{ }^{*} T} d_{i}^{*}+d_{i}{ }^{T} d_{i}-2 d_{i}^{{ }^{* T}} R d_{i}\right)
$$

)

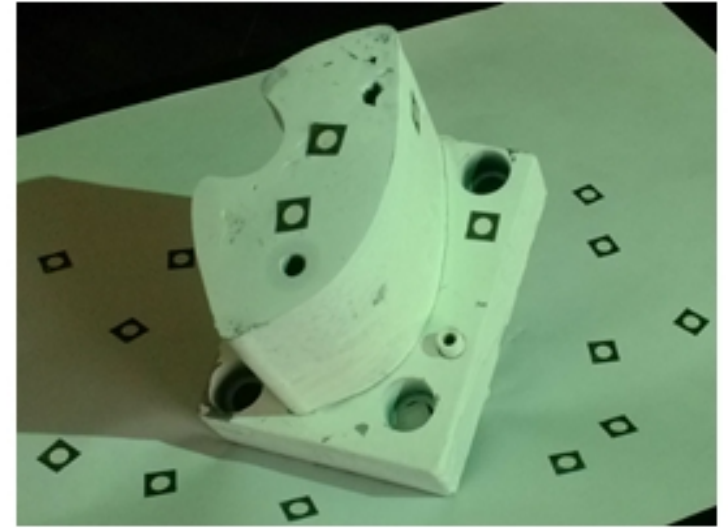

Figure 3 The part after processed

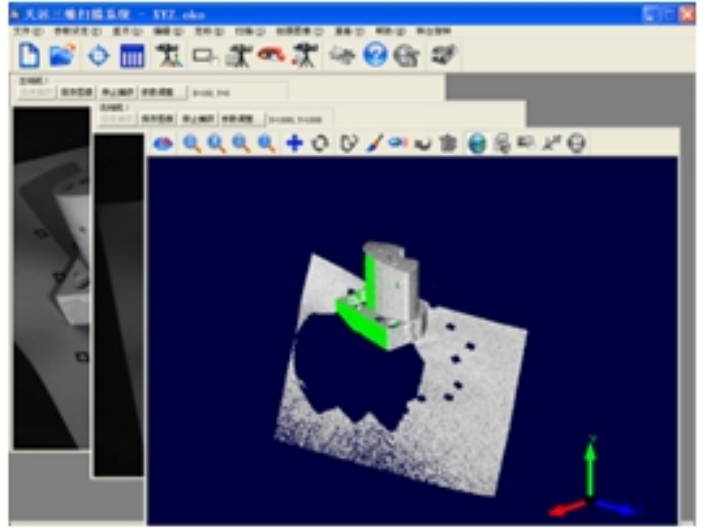

Figure 4 Twice scan obtained data

Then the multi view registration problem divided into two parts:

(1)Get a rotation transformation matrix $\bar{R}$ that could satisfy the requirement of least squares function $\mathrm{F}$;

(2)On the basis of $\bar{T}=c^{*}-\bar{R} c$ to solving the transformation matrix $\bar{T}$.

In this paper, by using the least square fitting method for the 3D scans of parts. After processing the parts as shown in Figure 3,based on symbolic point data as the benchmark for the least squares fitting splicing, twice scan data obtained as shown in figure 4 , Green part is the result of the second scan and error of joining together between two scan less than $0.02 \mathrm{~mm}$.

\section{surface reconstruction}

The results obtained through multiple scan as shown in figure 5,Aiming at the structural characteristics of stamping die parts, this article uses the method of reconstruction of curved surface after fitting characteristic curve .First, create a section curve based on point cloud data; then use tensile, multi sections surface, sweep, filling etc which from generative surface design module to create the surface and obtained the parts which through the CATIA subsequent processing as shown in Figure 6.

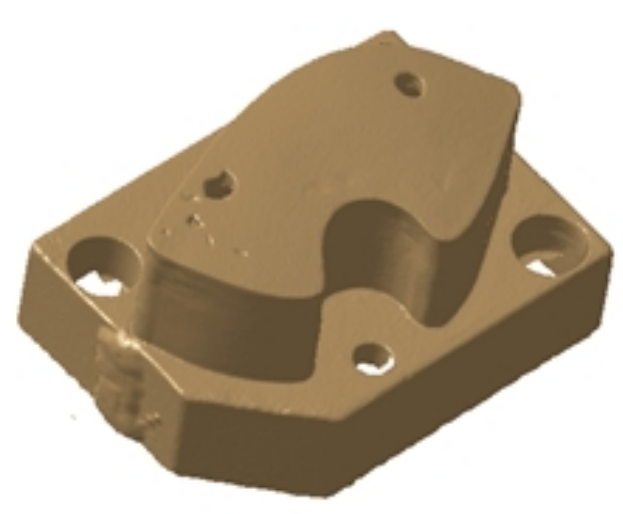

Figure 5 Scan point cloud data

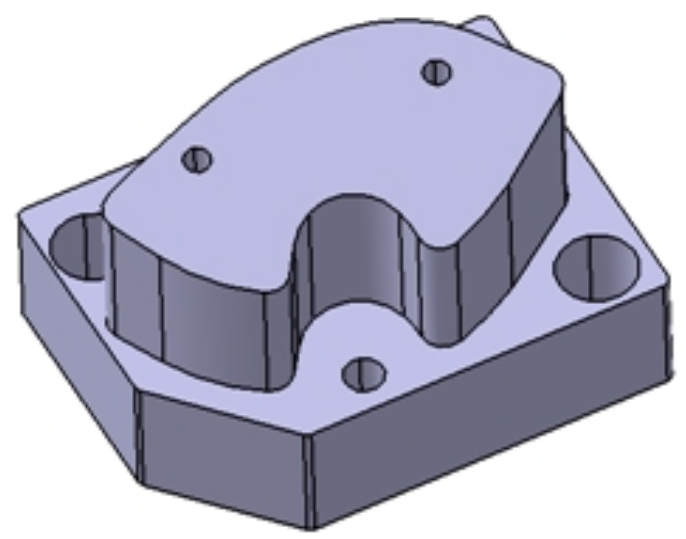

Figure 6 The reconstruction 3D model 


\section{Summary}

In this paper, aiming at the Stamping die parts which composed of free surface and use the method of non-contact scanning of binocular vision, the multi view registration technology obtained the 3-D shape by the free surface and three-dimensional point cloud data; secondly, parts of reverse modeling study was made by surface reconstruction technology and the results show that the method is suitable for the composed of complex curved surface parts of reverse design, convenient operation, high precision.

\section{References}

[1] Hocken R J ,Raja J,Babu U,Sampling Issures Incoordinate Metrology , Manufacturing Review ,1993,6(4):282-294.

[2] Miyin M, Kruth JP. Parametrisation of randomly measured points for the least square fittingodf B-spline curves and surface . Computer Aided Design,1995, 27:267-290.

[3] Lewis B A, Robinson J S. Triangulation of planar Region with Applcations , The ComputerJournal .2008,21(4):324-332.

[4] Andre Aman Claudet, Analysis of three dimenstional measurement data and CAD models PHD thesis ,Georgia Institute of Technology , 2001(3):30-80. 